\title{
Java Ranger at SV-COMP 2020 (Competition Contribution)
}

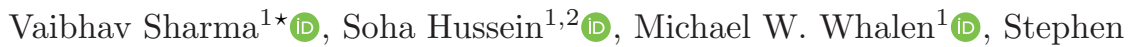 \\ McCamant $^{1}$, and Willem Visser ${ }^{3}$ (1) \\ 1 University of Minnesota, Minneapolis, MN, USA \\ \{vaibhav, husse200, mwwhalen, smccaman\}@umn.edu \\ 2 Ain Shams University, Cairo, Egypt \\ soha.hussien@cis.asu.edu.eg \\ 3 Stellenbosch University, Stellenbosch, South Africa \\ visserw@sun.ac.za

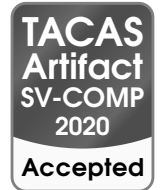

\begin{abstract}
Path-merging is a known technique for accelerating symbolic execution. One technique, named "veritesting" by Avgerinos et al. uses summaries of bounded control-flow regions and has been shown to accelerate symbolic execution of binary code. But, when applied to symbolic execution of Java code, veritesting needs to be extended to summarize dynamically dispatched methods and exceptional control-flow. Such an extension of veritesting has been implemented in Java Ranger by implementing as an extension of Symbolic PathFinder, a symbolic executor for Java bytecode. In this paper, we briefly describe the architecture of Java Ranger and describe its setup for SV-COMP 2020.
\end{abstract}

\section{Approach}

Symbolic execution is a well-known program analysis technique that has been applied to many applications such as test generation [3,7], equivalence checking $[6,8]$, and vulnerability finding [13]. However, when applied to large software, symbolic execution can suffer from scalability challenges caused by path explosion. Path-merging techniques such as veritesting [1] and dynamic state merging [4] help alleviate these scalability limitations. In particular, veritesting attempts to construct a static summary of a multi-path region and use it. Veritesting has been shown to significantly accelerate symbolic execution of binary code. Given that a large amount of software in use today is still written in Java, it is desirable to bring the benefits of veritesting to symbolic execution of Java as well. However, features such as dynamic dispatch make path-merging for Java code challenging [11]. The summary of a multi-path region that contains a dynamically-dispatched method call can only be constructed if the method to be called can also be summarized. Java Ranger (JR) extends the current stateof-the-art path-merging ideas presented by Avgerinos et al. [1] by first building static summaries which are later transformed using runtime information such as

\footnotetext{
* Jury Member
}

(C) The Author(s) 2020

A. Biere and D. Parker (Eds.): TACAS 2020, LNCS 12079, pp. 393-397, 2020.

https://doi.org/10.1007/978-3-030-45237-7_27 
the dynamic type of an object reference used for accessing a field. Java Ranger is built as an extension to Symbolic PathFinder (SPF) [5].

\section{Architecture}

Java Ranger is implemented as an SPF listener that watches for symbolic branch conditions in branching instructions. On encountering a symbolic branch instruction, JR attempts to create a summary for the multi-path region that begins at that branch instruction and ends at its exit points. A multi-path region is a region of code that begins at a branch instruction with a symbolic branch condition. An exit point of a multi-path region is either (1) the first program location in a control-flow path through the multi-path region which could not be summarized, or (2) the location of the immediate post-dominator of the multi-path region. This mechanism is also explained by Sharma et al. [12] in Figure 4.

\section{Strengths And Weaknesses}

Since JR improves scalability limitations of symbolic execution, its strength can only be observed when running it over large software. However, JR falls back to vanilla symbolic execution when it finds no opportunity for path-merging. SV-COMP 2020 had 416 verification tasks in the Java track. More information on SV-COMP 2020 can be found in its competition report [2]. JR instantiated at least one static summary on 96 different benchmarks of the 416 benchmarks. The summary for a multi-path region can be instantiated more than once on each benchmark because it is possible that the symbolic executor will encounter the same multi-path region more than once while running the benchmark. In total, JR instantiated 356 unique summaries. The total number of instantiated summaries used by JR was 20,182. JR also inlined a method summary a total of 62,857 times while instantiating these summaries.

JR also had a "unknown" conclusion on 40 of the 416 SV-COMP 2020 verification tasks. 22 of the 40 were caused due to our JR configuration which turned off support for symbolic strings because we found SPF's support for solving string constraints was not stable. 9 "unknown" conclusions were reached due to missing support for symbolic array lengths in multi-dimensional arrays. 8 of the 40 occurred due to a timeout. The last "unknown" result occurs in the equivalence check verification task in the ApacheCLI benchmark due to JR's use of a depth limit.

We made use of two depth limit parameters in SV-COMP 2020. The first was a limit on the exploration depth of our baseline symbolic executor, SPF. The second was a depth limit on the recursive depth to which our method summaries would be inlined. While we wished to avoid the use of any such limit, we found similar kinds of limits were used by many participanting tools in SVCOMP 2019. It is common to use some kind of limitation when applying symbolic execution tools in practice, since they can get bogged down by path explosion or related problems, and path-merging helps with but does not eliminate this issue. 
The Java verification category of SV-COMP 2020 did not score a tool's answer differently if it used a depth limit for producing that answer. Instead, the use of depth limit is reflected in each tool's score only if it caused the tool to produce an incorrect answer. We describe these depth limits and JR's configuration options in the following section.

\section{Tool Setup and Configuration}

Java Ranger's setup is very similar to the setup used by SPF. Since Java Ranger is simply an extension of SPF, the Java Ranger directory can be specified as a valid jpf-symbc extension of JPF. A JR configuration requires the following additions.

veritestingMode $=\langle 1-5\rangle$

veritestingMode specifies the path-merging features to be enabled with each higher number adding a new feature to the set of features enabled by the previous number. Setting veritestingMode to 1 runs vanilla SPF. Setting it to 2 enables path-merging for multi-path regions with no method calls and a single exit point. Setting it to 3 adds path-merging for multi-path regions that make method calls where the method can be summarized by Java Ranger. Setting it to 4 adds pathmerging for multi-path regions with more than one exit point caused due to exceptional behavior and unsummarized method calls. Setting it to 5 adds pathmerging for summarizing return instructions in multi-path regions by treating them as an additional exit point.

performanceMode $=$ <true or false>

Setting performanceMode to true causes Java Ranger to minimize the number of solver calls to check the feasibility of the path condition when summarizing a multi-path region with multiple exit points.

TARGET_CLASSPATH_WALA=<classpath of target code>

Java Ranger needs this variable to be set up as environment variable. It is not part of the .jpf configuration file. This environment variable tells Java Ranger where it should be expecting to find code that needs to be statically summarized.

jitAnalysis=<true or false>

When turned on (the default value), this option causes JR to summarize multipath regions when it encounters them. When turned off, JR attempts to summarize all multi-path regions reachable in a statically-computed interprocedural call graph up to a configurable limit.

recursiveDepth=<an integer value>

This option forces JR to restrict inlining of method summaries up to the value provided for this option. We set this parameter to 12 for SV-COMP 2020.

The following option is a JPF [14] configuration option which we also used for SV-COMP 2020.

search.depth_limit=<an integer value>

This option forces JPF to restrict its exploration to the depth provided as the value for this option. JPF constructs a tree of possible choices and explores the tree in a heuristic order, depth-first by default. Since JR is built as an extension 
to SPF, which is in turn built as an extension to JPF, we were able to restrict JR's exploration of choices using this option. We set this parameter to the value 13 for SV-COMP 2020.

\section{Software Project and Contributors}

Java Ranger is an extension of SPF. It is maintained on GitHub [9]. The version of Java Ranger that participated in Sv-COMP 2020 is publicly available [10]. For more information, please contact the authors of this paper.

\section{Acknowledgments}

The research described in this paper has been supported in part by the National Science Foundation under grant 1563920.

\section{References}

1. Avgerinos, T., Rebert, A., Cha, S.K., Brumley, D.: Enhancing Symbolic Execution with Veritesting. In: Proceedings of the 36th International Conference on Software Engineering. pp. 1083-1094. ICSE 2014, ACM, New York, NY, USA (2014)

2. Beyer, D.: Advances in automatic software verification: SV-COMP 2020. In: Proc. TACAS (2). LNCS 12079, Springer (2020), https://www.sosy-lab.org/research/ pub/2020-TACAS.Advances_in_Automatic_Software_Verification_SV-COMP_ 2020.pdf

3. Godefroid, P., Klarlund, N., Sen, K.: DART: Directed Automated Random Testing. In: Proceedings of the 2005 ACM SIGPLAN Conference on Programming Language Design and Implementation. pp. 213-223. ACM, New York, NY, USA (2005)

4. Kuznetsov, V., Kinder, J., Bucur, S., Candea, G.: Efficient State Merging in Symbolic Execution. In: PLDI. pp. 193-204. PLDI '12, ACM, New York, NY, USA (2012)

5. Păsăreanu, C.S., Visser, W., Bushnell, D., Geldenhuys, J., Mehlitz, P., Rungta, N.: "Symbolic PathFinder: Integrating Symbolic Execution With Model Checking For Java Bytecode Analysis". Automated Software Engineering 20(3), 391-425 (Sep 2013)

6. Ramos, D.A., Engler, D.R.: Practical, Low-effort Equivalence Verification of Real Code. In: Proceedings of the 23rd International Conference on Computer Aided Verification. pp. 669-685. CAV'11, Springer-Verlag, Berlin, Heidelberg (2011)

7. Sen, K., Marinov, D., Agha, G.: CUTE: A Concolic Unit Testing Engine for C. In: Proceedings of the 10th European Software Engineering Conference Held Jointly with 13th ACM SIGSOFT International Symposium on Foundations of Software Engineering. pp. 263-272. ESEC/FSE-13, ACM, New York, NY, USA (2005)

8. Sharma, V., Hietala, K., McCamant, S.: Finding Substitutable Binary Code By Synthesizing Adaptors. In: 11th IEEE Conference on Software Testing, Validation and Verification (ICST) (Apr 2018)

9. Sharma, V., Hussein, S., Whalen, M.W., McCamant, S., Visser, W.: Java Ranger. https://github.com/vaibhavbsharma/java-ranger (2019-2020)

10. Sharma, V., Soha, Michael, Stephen, Willem: Java Ranger at SV-COMP 2020 (Feb 2020). https://doi.org/10.5281/zenodo.3678718 
11. Sharma, V., Whalen, M.W., McCamant, S., Visser, W.: Veritesting Challenges in Symbolic Execution of Java. In: Java PathFinder Workshop (Jan 2018)

12. Sharma, V., Whalen, M.W., McCamant, S., Visser, W.: Veritesting challenges in symbolic execution of Java. SIGSOFT Softw. Eng. Notes 42(4), 1-5 (Jan 2018). https://doi.org/10.1145/3149485.3149491

13. Stephens, N., Grosen, J., Salls, C., Dutcher, A., Wang, R., Corbetta, J., Shoshitaishvili, Y., Kruegel, C., Vigna, G.: Driller: Augmenting Fuzzing Through Selective Symbolic Execution. In: Network and Distributed System Security Symposium (NDSS) (2016)

14. Visser, W., Havelund, K., Brat, G., Park, S., Lerda, F.: Model checking programs. Automated Software Engineering 10(2), 203-232 (Apr 2003). https://doi.org/10.1023/A:1022920129859

Open Access This chapter is licensed under the terms of the Creative Commons Attribution 4.0 International License (http://creativecommons.org/licenses/by/4.0/), which permits use, sharing, adaptation, distribution and reproduction in any medium or format, as long as you give appropriate credit to the original author(s) and the source, provide a link to the Creative Commons license and indicate if changes were made.

The images or other third party material in this chapter are included in the chapter's Creative Commons license, unless indicated otherwise in a credit line to the material. If material is not included in the chapter's Creative Commons license and your intended use is not permitted by statutory regulation or exceeds the permitted use, you will need to obtain permission directly from the copyright holder. 